\title{
AVALIAÇÃO DE LINHAS DE BENEFICIAMENTO E IMPACTOS DE QUEDA NA QUALIDADE DE CAQUI 'RAMA FORTE"
}

\author{
SILVIA R. DE T. VALENTINI ${ }^{1}$, MARCOS D. FERREIRA ${ }^{2}$, MÁRCIA E. ATARASSI ${ }^{3}$, \\ ELIANE A. BENATO ${ }^{4}$
}

\begin{abstract}
RESUMO: O manuseio inadequado e excessivo de frutas e de hortaliças causa alta incidência de danos físicos e, consequentemente, perdas pós-colheita. $\mathrm{O}$ objetivo deste trabalho foi avaliar a magnitude de impacto em linhas de beneficiamento e classificação para caqui "Rama Forte" e determinar, em laboratório, seus limites de impactos. Para a avaliação dos pontos críticos, foi utilizada uma esfera instrumentada de $76 \mathrm{~mm}$ de diâmetro (Techmark, Inc., Lansing, EUA), que registrou a magnitude dos impactos em sete equipamentos distintos de quatro unidades de beneficiamento. A determinação de danos físicos foi feita em testes laboratoriais, nos quais a queda dos frutos foi relacionada com a magnitude de impacto, incidência de danos físicos e perdas na qualidade do fruto. Nas linhas de beneficiamento, os valores de impactos encontrados variaram de 21 a 87 G nos pontos de transferência, e a maioria dos impactos registrados (acima de 94\%) durante a passagem pela linha foram de valores de até $50 \mathrm{G}$. Alturas de queda a partir de $20 \mathrm{~cm}$ causaram aumento na perda de massa do caqui no sexto dia de armazenamento, em temperatura ambiente. As quedas de 20 e $30 \mathrm{~cm}$ causaram maior escurecimento da casca (menores valores de L), associada à redução da intensidade da cor (chroma). O impacto não causou efeito nas características químicas da polpa do fruto.
\end{abstract}

PALAVRAS-CHAVE: Diospyros kaki L., esfera instrumentada, aceleração (G), danos físicos.

\section{PACKING LINE EVALUATION FOR DROP IMPACTS ON PERSIMMON QUALITY “RAMA FORTE”}

\begin{abstract}
Excessive and inadequate handling of fruits and vegetables provides high incidences of physical damage, consequently, post harvest losses. The main goal of this work was to evaluate the impact magnitude in persimmon packing lines, "Rama Forte", and to determine, at the laboratory, its impact limits. For evaluating the critical points it was used an instrumented sphere of $76 \mathrm{~mm}$ of diameter (Technmark, Inc, Lansing, USA), which registered the impact magnitude in seven distinctive impact lines located in four packing houses. For determining physical damages, tests were carried out at the laboratory, where fruit drop was related to impact magnitude, physical damage incidence and fruit post harvest losses. At the packing lines, the values found varied from 21 to $87 \mathrm{G}$ on the transfer points and the majority of registered impacts (over 94\%) were down 50G. Drops from $20 \mathrm{~cm}$ caused an increase in weight losses after six days of storage at room temperature. Drops from 20 and $30 \mathrm{~cm}$ caused skin darkness (low L values), associated to a decrease in color intensity (chroma). Impact drop did not affect pulp fruit chemical features.
\end{abstract}

KEYWORDS: Diospyros kaki L., instrumented sphere, acceleration (G), physical damage.

\footnotetext{
${ }^{1}$ Pesquisador científico, Instituto de Tecnologia de Alimentos, Av. Brasil, 2880, Campinas - SP, valentini@ital.sp.gov.br

${ }^{2}$ Pesquisador A, Embrapa Instrumentação Agropecuária, Caixa Postal 741, São Carlos - SP, Fone: (0xx16-2107-2804), Pesquisador Colaborador, Faculdade de Engenharia Agrícola, Unicamp, Campinas - SP, marcosferreira@cnpdia.embrapa.br

${ }^{3}$ Engenheira Agrícola, FEAGRI-UNICAMP, Av. Cândido Rondon, 501, Campinas - SP, atarassi@ yahoo.com.br

${ }^{4}$ Pesquisador científico, Instituto de Tecnologia de Alimentos, Av. Brasil, 2880, Campinas - SP, benato@ital.sp.gov.br

Recebido pelo Conselho Editorial em: 31-8-2007

Aprovado pelo Conselho Editorial em: 7-8-2009
} 


\section{INTRODUÇÃO}

O caqui é originário da Ásia, sendo produzido em regiões de clima temperado e subtropical, e sua colheita é feita durante os meses de fevereiro a abril e mais tardiamente até junho (SATO \& ASSUMPÇÃO, 2002). Segundo a Fundação IBGE, em 2005, foram produzidas cerca de 165 mil toneladas de caqui, que corresponderam a $\mathrm{R} \$ 118,40$ milhões. A região Sudeste foi a maior produtora, com 107,8 mil toneladas. Segundo SILVA et al. (2005), a oferta do caqui no período de safra é muito maior que a demanda, devido ao baixo consumo no País, ao curto período de colheita, à alta perecibilidade dos frutos e ao mercado regionalizado. Assim, a solução para a queda de preços e perdas do produto seria investir em logística e práticas de pós-colheita, aumentando a qualidade do fruto e o período de oferta no mercado.

Os impactos sofridos pelos frutos durante a colheita e o beneficiamento podem causar danos internos e externos, que afetam a qualidade do produto comercializado. Assim, conhecer os limites para surgimento de danos físicos é importante para a realização de manuseio adequado, evitando-se prejuízos posteriores. Além de depreciar a qualidade e contribuir, muitas vezes, para a imediata perda física do produto, os danos mecânicos favorecem o rápido amadurecimento, senescência, deterioração e consequente redução da vida pós-colheita de frutos (MATTIUZ \& DURIGAN, 2001; MATTIUZ \& DURIGAN, 2003; DAREZZO et al., 2003; MENCARELLI et al., 1996). Estes autores, ao estudarem a influência do impacto em frutas (goiabas, kiwis) e hortaliça (alface), observaram alterações na curva de respiração e de etileno, contribuindo para o rápido amadurecimento e/ou senescência do tecido vegetal. No Brasil, as etapas de beneficiamento e classificação do caqui "Rama Forte" são, em geral, realizadas em equipamentos projetados para outras frutas. Portanto, faz-se necessário que o manuseio pós-colheita seja adequado às características físicas específicas do produto. Todavia, não existem informações sobre a sensibilidade do caqui ao impacto nas condições brasileiras, que seria o primeiro passo para desenvolver e avaliar equipamentos que não prejudiquem a qualidade do fruto.

Danos por impacto na pós-colheita podem ocorrer quando os frutos são submetidos a quedas altas ou colidem com superfícies não protegidas ou com outros frutos. A severidade dos danos causados por impactos em uma linha de classificação pode ser reduzida, diminuindo-se a altura de queda entre as etapas, por meio da utilização de protetores nas superfícies, que podem dissipar a força de impacto ou também mantendo-se as áreas entre as etapas completas de frutas (HYDE \& ZHANG, 1992). O número de impactos também pode ser reduzido, diminuindo-se o número de quedas e pontos de transferência em uma linha de classificação. Nesse sentido, SARGENT et al. (1999) sugerem que a linha de beneficiamento e de classificação deve ser projetada preferencialmente em linha reta, sem desvios e curvas, e possuir alturas mínimas entre as etapas.

Para avaliar os pontos de injúrias em linhas de beneficiamento, podem-se utilizar umas esferas instrumentadas, que possuem dimensões, massa e formato aproximados da fruta a ser estudada, podendo variar desde menores dimensões $(20-30 \mathrm{~mm})$ até superiores a $90 \mathrm{~mm}$. A esfera é colocada junto ao produto e registra dados de impactos, mudança de velocidade e o intervalo entre impactos, que posteriormente são transferidos a um computador (ZAPP et al., 1990). A esfera instrumentada vem sendo utilizada em avaliações de linhas de beneficiamento de batatas (FERREIRA \& NETTO, 2005; HYDE et al., 1992), cebola (BAJEMA \& HYDE, 1995), laranjas (FERREIRA et al., 2006; MILLER \& WAGNER, 1991), maçãs (BROWN et al., 1990) e tomate (FERREIRA et al., 2005; SARGENT et al., 1992). Segundo GARCÍA-RAMOS et al. (2003), as características do impacto dependem de diferentes parâmetros: velocidade, altura de transferência, materiais de recobrimento e de como foram projetados os pontos de transferência.

Em laboratório, a avaliação de danos é feita simulando os impactos ocorridos na pós-colheita de frutas e hortaliças, por meio de sistema controlado de lançamento de frutos (a vácuo ou por um sistema eletromagnético), em superfície rígida ou recoberta com material emborrachado, reproduzindo a situação da linha de beneficiamento (FLUCK \& HALSEY, 1973; CHEN \& YAZDANI, 1991; BRUSEWITZ et al., 1991; SARGENT et al., 1992; MANESS et al., 1992). 
Os impactos podem gerar danos internos, devido a mudanças metabólicas originadas pela injúria sendo, em muitos casos, essas injúrias não percebidas externamente. Assim, foram desenvolvidas algumas metodologias para mensurar o dano físico causado. CHEN \& YAZDANI (1991) calcularam o volume do dano causado em maçãs, cortando-se o fruto no centro da área afetada e medindo-se o diâmetro e a profundidade do dano. SARGENT et al. (1992) e MORETTI et al. (1998) concluíram que a medição de danos internos mostrou-se adequada para a avaliação de impactos em tomate. Contudo, BOLLEN (2006) relata que ainda existe dificuldade de relacionar as informações obtidas em laboratório para predizer os danos causados durante o manuseio. Mensurações mais exatas têm ocorrido quando se estabelece relação entre a energia utilizada no impacto e o nível de dano ocorrido. Esse mesmo autor propõe dois métodos para a mensuração da incidência de danos físicos: o primeiro relaciona-se à amostragem de frutos durante o manuseio e a quantificação dos danos físicos, e a segunda refere-se à medição das forças que agem no sistema e correlacioná-las aos níveis de danos físicos que podem ser esperados.

Este trabalho teve como objetivo avaliar a magnitude de impacto nas linhas de beneficiamento, por meio de esfera instrumentada, e analisar em laboratório a influência de impacto na qualidade do caqui "Rama Forte" submetido à queda em superfície rígida, em três diferentes alturas de queda.

\section{MATERIAL E MÉTODOS}

Os ensaios foram divididos em duas etapas, sendo a primeira realizada em unidades de beneficiamento e classificação, e a segunda, em laboratório. A seguir, descrição das duas etapas.

\section{Primeira etapa - Avaliação da magnitude de impacto nas linhas de beneficiamento}

Nessa etapa, avaliou-se a magnitude de impactos nos pontos de transferência das linhas de classificação de caquis, no total de quatro unidades e sete equipamentos distintos. A seguir, breve descrição de cada equipamento avaliado.

A primeira unidade (1) analisada era composta de duas linhas de beneficiamento e classificação. Em ambas, a alimentação da linha e a classificação eram feitas manualmente. A primeira linha possuía 9,56 m de comprimento e cinco pontos de transferência, sendo composta de uma bancada de entrada, seguida de roletes transportadores e escovas de polimento de crina de cavalo. Na saída do transporte, o desnível era de $14 \mathrm{~cm}$, enquanto na saída do polimento este valor era de $8 \mathrm{~cm}$. Após o polimento, havia duas derivações para esteiras que levavam os frutos a bancadas acolchoadas de classificação: uma destinada para o caqui "Rama Forte" (linha A) e a outra para o caqui "Taubaté" (linha B). Na entrada da classificação manual, os frutos eram submetidos à queda de até $5 \mathrm{~cm}$, e as bancadas eram inclinadas, com $24 \mathrm{~cm}$ de desnível. A segunda linha (C) possuía 12,54 m de comprimento e cinco pontos de transferência. Era destinada para o caqui "Rama Forte", sendo composta de uma bancada de recebimento, esteira de transporte, polimento com escova de crina de cavalo e bancada acolchoada de classificação manual. Na saída do transporte, o desnível era de $33 \mathrm{~cm}$, enquanto na saída do polimento era de $11 \mathrm{~cm}$. As bancadas de classificação eram inclinadas, com $24 \mathrm{~cm}$ de desnível.

A segunda unidade (2) possuía duas linhas (D e E) de beneficiamento e de classificação de caqui "Rama Forte". A linha (D) apresentava 7,70 m de comprimento e quatro pontos de transferência. No começo da linha, havia uma bancada de recebimento dos frutos, que eram colocados manualmente na linha de polimento com escovas de crina de cavalo. Na saída dessa etapa, havia desnível de $8 \mathrm{~cm}$. Em seguida, uma esteira de lona levava os frutos para bancadas acolchoadas de classificação manual. Os desníveis nessa parte da linha eram de $22 \mathrm{~cm}$ entre a esteira e a bancada, e na bancada era de $30 \mathrm{~cm}$. A linha (E) possuía 14,75 m e quatro pontos de transferência. Nela havia uma bancada de entrada, seguida por roletes transportadores, escovas de polimento de crina de cavalo, esteiras e bancada acolchoada de classificação. Na saída do transporte e na saída das escovas de polimento da linha E, o desnível era de $19 \mathrm{~cm}$. 
A unidade 3 era composta de uma linha de beneficiamento e de classificação (linha F), com $20,90 \mathrm{~m}$ de comprimento e seis pontos de transferência. No recebimento, havia um virador de caixas que colocava as frutas em uma esteira de rolos transportadores, que levavam para um conjunto de escovas. Saindo do polimento, havia discos de borracha para alinhar os caquis antes da fase de classificação por peso. Por final, as frutas seguiam para as bancadas de embalagem. Na saída do polimento, a altura de queda era de $15 \mathrm{~cm}$ sobre uma chapa de metal. Na saída dos discos de borracha, havia desnível de $5 \mathrm{~cm}$, e na saída da classificação, a altura de queda era de $8 \mathrm{~cm}$ sobre uma esteira de lona.

A quarta unidade possuía uma linha de beneficiamento de caqui "Rama Forte", com duas derivações para a classificação dos frutos. Na etapa de recebimento, os frutos eram depositados em uma esteira de roletes e, em seguida, submetidos ao polimento em escovas de crina de cavalo. Após a escovação, ocorria uma seleção para a retirada de defeitos, e depois a linha de beneficiamento dividia-se em duas (linhas $\mathrm{G}$ e $\mathrm{H}$ ). Antes da entrada no classificador, os produtos eram acondicionados em caixas de recebimento, semelhantes às encontradas no início da linha, proporcionando queda e interrupção de fluxo. Um funcionário retirava manualmente os frutos dessa caixa para a classificação, que ocorria por meio de equipamentos mecânicos de pesagem de frangos adaptados para caquis. Os desníveis encontrados na linha foram de $38 \mathrm{~cm}$ na entrada, $35 \mathrm{~cm}$ na saída do recebimento e $3 \mathrm{~cm}$ na saída do polimento. A altura de queda nas caixas posicionadas antes da classificação era de $23 \mathrm{~cm}$ na linha $\mathrm{G}$ e de $29 \mathrm{~cm}$ na linha $\mathrm{H}$. Ao colocar o fruto no equipamento de classificação, os frutos sofriam queda de até $25 \mathrm{~cm}$ na linha $\mathrm{G}$ e de $30 \mathrm{~cm}$ na linha H. Nas duas linhas, a saída da classificação tinha desnível de $25 \mathrm{~cm}$.

A esfera instrumentada (Techmark, Inc., Lansing, EUA) de $76 \mathrm{~mm}$, equipamento plástico com registrador de aceleração e limite para o nível de impacto variando entre $15 \mathrm{G}$ e $500 \mathrm{G}$, foi colocada juntamente com os frutos na etapa de recebimento de cada linha de beneficiamento, que funcionava com sua capacidade normal, seguindo o fluxo dos mesmos até a etapa de classificação. O tempo de permanência da esfera instrumentada em cada etapa, assim como o tempo para completar o percurso total e os pontos de transferência foram monitorados por meio de cronômetro de precisão.

As medições no percurso total da linha de classificação foram repetidas cinco vezes. Após os testes, a esfera instrumentada era removida, e os dados transferidos, e analisada em computador, avaliando a magnitude dos impactos a que os frutos foram submetidos nos pontos de transferência das linhas de beneficiamento. Dados médios de impacto, obtidos nos pontos de transferência de cada unidade de beneficiamento avaliada, foram correlacionados com a aceleração máxima (AM) $\left(\mathrm{G}=9,81 \mathrm{~m} \mathrm{~s}^{-2}\right)$.

\section{Segunda etapa - Efeito do impacto de queda na qualidade físico-química do caqui "Rama Forte"}

Caquis da variedade "Rama Forte" foram colhidos manualmente no estádio de maturação fisiológica e transportados para o laboratório da Faculdade de Engenharia Agrícola, da Universidade Estadual de Campinas, onde foram selecionados para a retirada de frutos com danos provenientes de campo. Os pedúnculos dos frutos foram cortados, evitando-se, assim, injúrias a outros frutos. Frutos destanizados foram submetidos à queda em três diferentes alturas: 10; 20 e $30 \mathrm{~cm}$, em superfície rígida (chapa metálica $2 \mathrm{~mm}$ ). Foram considerados como tratamento-controle caquis destanizados não submetidos à queda, sendo utilizados 40 frutos por tratamento.

A determinação da incidência para danos físicos foi realizada em equipamento, seguindo modelo descrito por MAGALHÃES et al. (2007). Esse equipamento não permite aceleração nem rotação do fruto no momento do lançamento, sendo composto pelos seguintes itens: aspirador de pó portátil, com 1.000 Watts de potência, registro de esfera PVC de 1", mangueira de plástico com $34 \mathrm{~mm}$ de diâmetro, suporte de lançamento de chapa de aço-carbono com $60 \mathrm{~cm}$ de altura, ajuste regulador da altura de queda, bocal para segurar os frutos constituído por uma ventosa de plástico com uma incisão circular no centro para a passagem do vácuo, superfície rígida de aço-carbono de $5 \mathrm{~mm}$ de espessura, onde ocorreu o impacto com o fruto. Na placa de aço, foi aplicada uma camada 
de pó de giz para marcar a área de impacto do fruto, que era delimitada por caneta permanente no fruto e, em seguida, limpa para retirar os resíduos de giz. Após a aplicação dos tratamentos, os frutos foram armazenados em câmara com temperatura média de $24^{\circ} \mathrm{C}$, por seis dias.

No $1^{\mathrm{o}} ; 3^{\mathrm{o}}$ e $6^{\mathrm{o}}$ dias de armazenamento, foram feitas avaliações de cor, perda de massa, incidência de danos físicos e análises químicas da polpa da fruta. A coloração foi medida em 20 frutos, em duas posições marcadas, por meio de colorímetro Hunter Lab, com miniscan XE Plus, escala CIELAB ( $\left.\mathrm{L}^{*}, \mathrm{a}^{*}, \mathrm{~b}^{*}\right)$, e os parâmetros avaliados foram a luminosidade ( $\left.\mathrm{L}^{*}\right)$ e a cromaticidade (Chroma). De acordo com TREVISAN et al. (2004), nesse sistema de representação de cor, os valores $\mathrm{L}^{*}, \mathrm{a}^{*} \mathrm{e} \mathrm{b}^{*}$ descrevem a uniformidade da cor no espaço tridimensional, em que o valor $\mathrm{L}^{*}$ corresponde à escala escuro-claro (valor zero para a cor preta e 100 para a branca). Os valores de $\left(\mathrm{a}^{*}\right)$ correspondem à escala do verde ao vermelho ( $\mathrm{a}^{*}$ negativo representa o verde, e $\mathrm{a}^{*}$ positivo, o vermelho), e os valores de $\left(b^{*}\right)$ correspondem à escala do azul ao amarelo ( $b^{*}$ negativo para o azul, e b* positivo para o amarelo). A cromaticidade representa a saturação ou intensidade da cor, em que o valor zero indica cor impura e 60 cor pura, sendo calculada pela equação Chroma $=$ $(a * 2+b * 2)^{1 / 2}$.

A perda de massa foi avaliada juntamente com a coloração, sendo expressa em percentagem, em relação ao primeiro dia de armazenamento. A incidência de danos físicos foi avaliada para cada tratamento em nove caquis, em relação ao amadurecimento do fruto, por meio do índice de adstringência e a percentagem de frutos com danos internos visíveis. O caqui foi cortado longitudinalmente e feita a impressão das metades do fruto em filtro de papel impregnado com $\mathrm{FeCl}_{3}$. $\mathrm{O}$ índice de adstringência foi dado de acordo com a coloração obtida no papel, seguindo a escala: 1 - não adstringente; 2 - ligeiramente adstringente a não adstringente; 3 - moderadamente adstringente; 4 - adstringente; 5 - muito adstringente (GAZIT \& LEVY, 1963). A quantidade de frutos com danos internos visíveis foi avaliada cortando-se o caqui ao meio e verificando a presença de descoloração da polpa, característico de dano físico. A percentagem de frutos com dano interno foi calculada em relação ao total de frutos analisados. As análises químicas realizadas foram de $\mathrm{pH}$ (potenciômetro), sólidos solúveis (refratômetro, com correção de temperatura para $20{ }^{\circ} \mathrm{C}$ ) e acidez titulável, expressas como ácido málico em g $100 \mathrm{~mL}^{-1}$ e realizadas segundo metodologia proposta por CARVALHO et al. (1990). Foi aplicado o teste de Tukey para a comparação de médias dos resultados (nível de confiança de 95\%), utilizando o programa Statgraphics.

\section{RESULTADOS E DISCUSSÃO}

\section{Avaliação da magnitude de impacto nas linhas de beneficiamento}

Na Figura 1, são apresentados os valores de impacto nas três linhas de beneficiamento e a classificação da unidade 1. Pode-se observar que, na linha $\mathrm{A}$, o nível máximo de impacto ocorreu no ponto de transferência 3, que se refere à saída da esteira, com impacto de 39,92 G. No entanto, observa-se que o impacto médio máximo na linha $\mathrm{B}$ ocorreu no ponto de transferência 1 , referente à entrada dos frutos na linha, apresentando aceleração média máxima igual a 65,18 G. Na linha C, o maior impacto ocorreu no ponto de transferência 2, quando os frutos entram nas escovas de polimento, com média de 67,34 G. Nas três linhas, a maioria dos impactos registrados foi de até $50 \mathrm{G}$ (média de $95 \%$ do total). Nas linhas A, B e C, a metade dos valores de impactos de até $50 \mathrm{G}$ ocorreu na etapa de polimento.

Na unidade 2, destinada ao beneficiamento e à classificação do caqui "Rama Forte", o maior impacto foi de $83,22 \mathrm{G}$, observado na linha $\mathrm{D}$, no ponto de transferência de entrada na bancada, enquanto, na linha E, o maior valor foi na saída das escovas, com 59,80 G (Figura 2). Nessas duas linhas, cerca de $94 \%$ dos impactos foram de até $50 \mathrm{G}$. Na linha D, 3,5\% dos impactos ocorreram na faixa de 50 a $100 \mathrm{G}$, enquanto a linha $\mathrm{E}$ apresentou 5,4\%, sendo a metade desses valores registrados no recebimento dos frutos dessas duas linhas. 


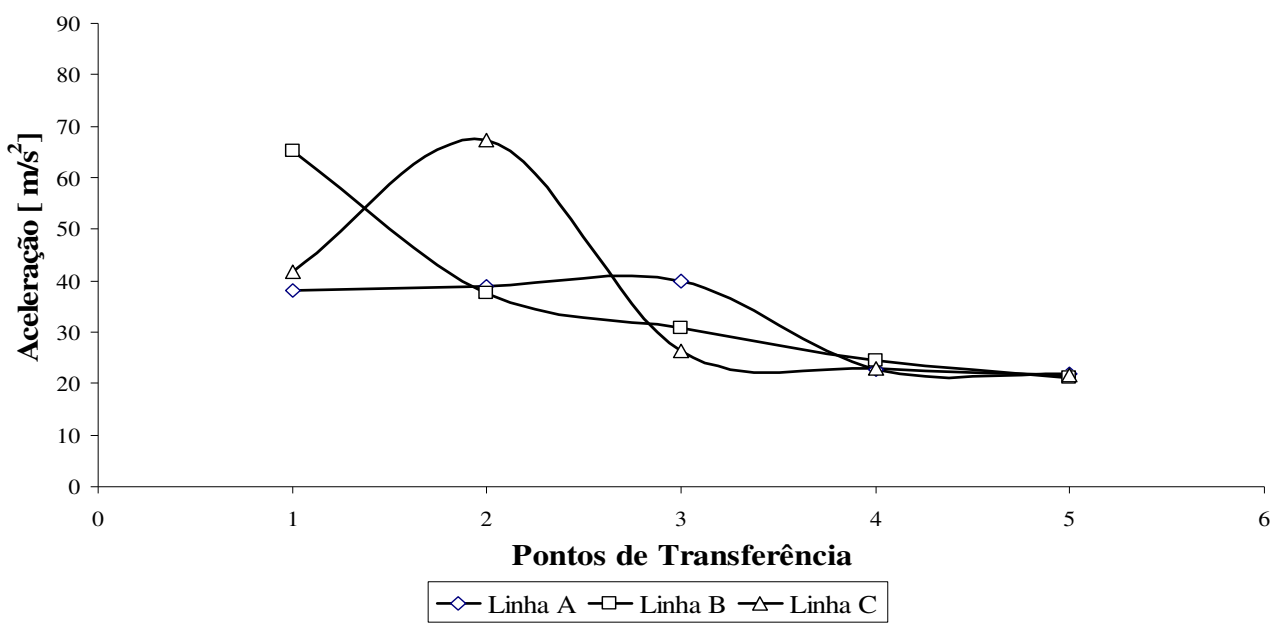

FIGURA 1. Acelerações médias (G) encontradas nas linhas de beneficiamento e de classificação da unidade 1 em seus pontos de transferência: 1 - Recebimento; 2 - Entrada nas escovas; 3 - Saída da esteira; 4 - Entrada na bancada; 5 - Queda final. Mean accelerations (G) found in the packing lines and classification of the unit 1 in its transfer points: 1 Receiving; 2 - Check strips; 3 - Output mat; 4 - Entering the countertop; 5 - Final fall.

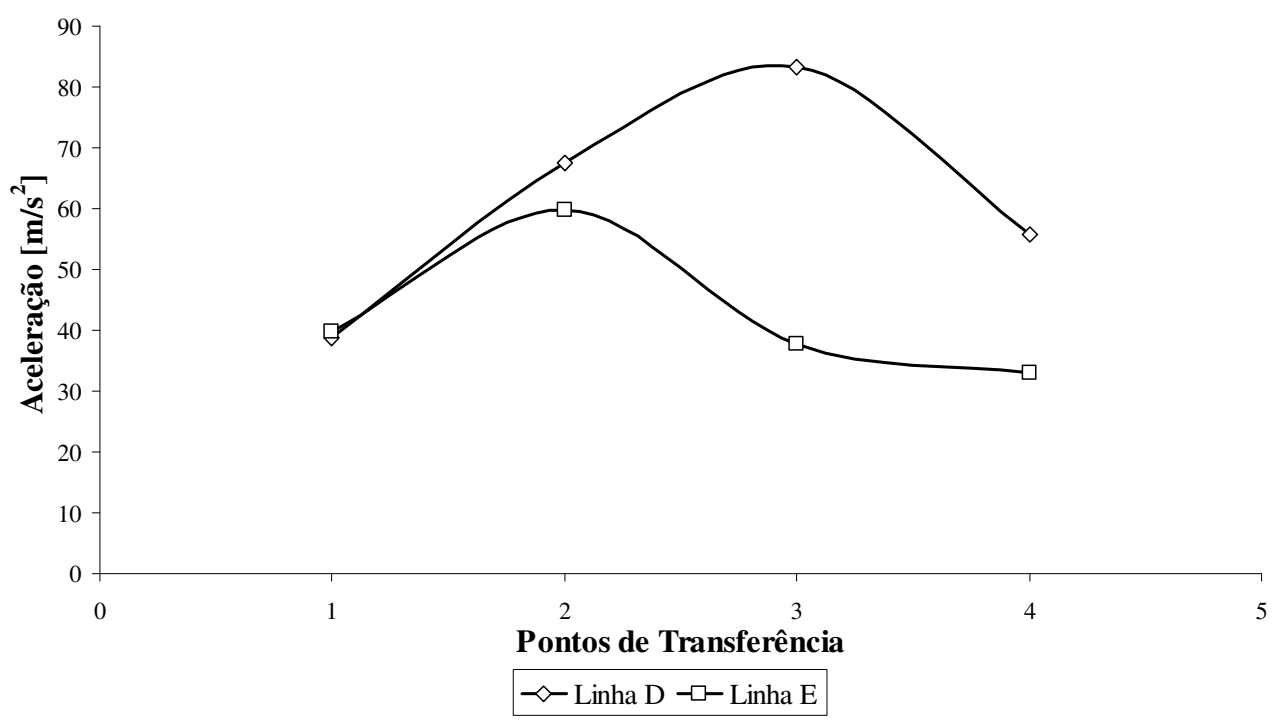

FIGURA 2. Acelerações médias (G) encontradas nas linhas de beneficiamento e de classificação da unidade 2 em seus pontos de transferência. Linha D: 1 - Recebimento; 2 - Saída das escovas; 3 - Entrada na bancada; 4 - Saída da bancada. Linha E: 1 - Recebimento; 2 Saída do transporte; 3 - Saída das escovas; 4 - Queda final. Mean accelerations (G) found in the packing lines and classification of the unit 2 in its transfer points. Line D: 1 - Receiving; 2 - Output from brushes; 3 - Entering the countertop; 4 Output from the sidelines. Line E: 1 - Receiving; 2 - Output transport; 3 - Output from brushes; 4 - Final fall. 
Observou-se que, no ponto de transferência de recebimento da unidade 3, ocorreu o maior valor de impacto da linha F, com valor de 63,68 G (Figura 3). Os impactos de até $50 \mathrm{G}$ corresponderam a 92,9\% do total registrado na linha, sendo que, desses, 29,1\% ocorreram na etapa de polimento. A faixa de impactos de 50 a $100 \mathrm{G}$ foi de $7,1 \%$, sendo $66,7 \%$ dos valores registrados entre os pontos de transferência 5 e 6 .

Na unidade 4, verificou-se o maior impacto na linha G, na entrada da classificação, com 81,06 G (Figura 3), enquanto na linha H, foi de $86,98 \mathrm{G}$ no ponto de transferência de entrada da escova. Nas duas linhas, os impactos concentraram-se em valores de até $50 \mathrm{G}$, com média de 93,6\% do total. Na faixa de 50 a $100 \mathrm{G}$, a linha $\mathrm{G}$ apresentou $6,9 \%$, enquanto na linha $\mathrm{H}$ a percentagem foi um pouco menor, com $5,2 \%$. As etapas de recebimento e de polimento foram as que concentraram a maior quantidade de valores, com 30 e $35,3 \%$ nas linhas $\mathrm{G}$ e $\mathrm{H}$, respectivamente.

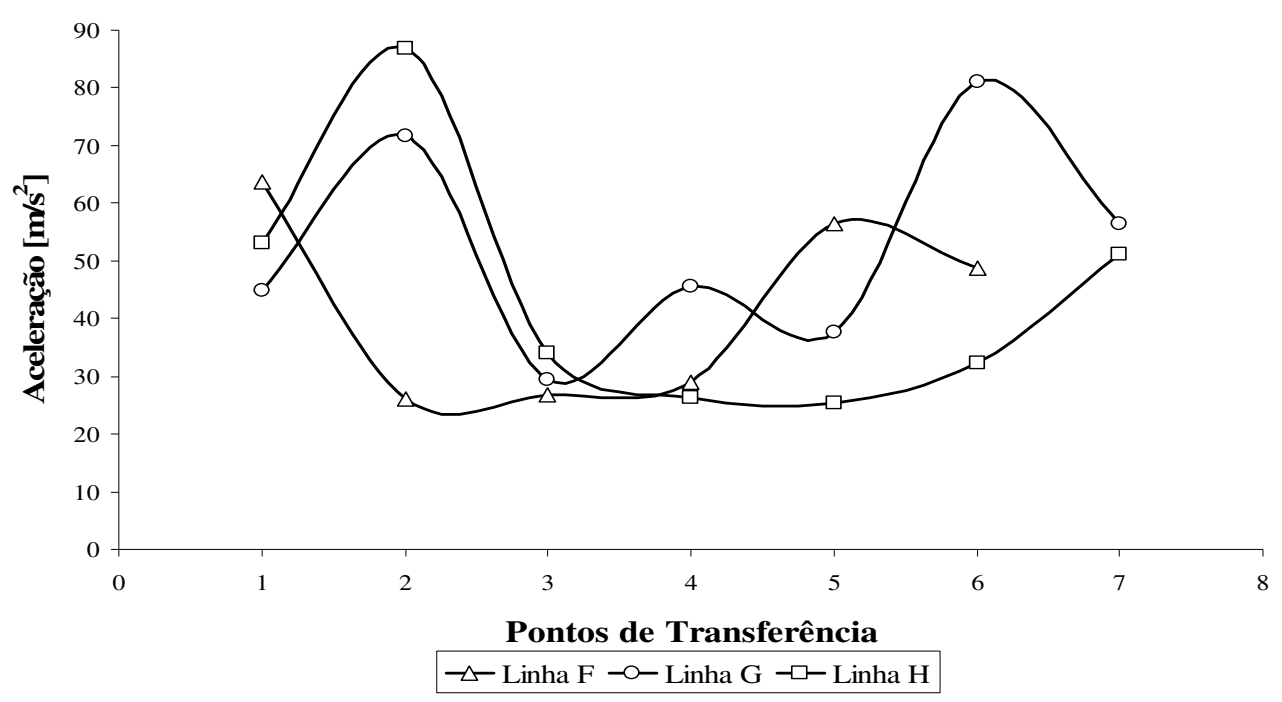

FIGURA 3. Acelerações médias (G) encontradas na linha de beneficiamento e de classificação F, G e H (unidades 3 e 4) em seus pontos de transferência: Linha F: 1 - Recebimento; 2 Entrada nas Escovas; 3 - Saída das escovas; 4 - Saída Disco; 5 - Saída classificação; 6 Queda na bancada. Linhas G e H: 1 - Recebimento; 2 - Entrada na escova; 3 - Saída da escova; 4 - Entrada na esteira; 5 - Esteira Bifurcação; 6 - Entrada na Classificação; 7 Saída da classificação. Mean accelerations (G) found in the processing and classification line F, $G$ and $H$ (units 3 and 4) in its transfer points: Line F: 1 Receiving; 2 - Check the brushes; 3 - Check out the brushes; 4 - Output disk; 5 Output rating; 6 - Decline bench. Lines G and H: 1 - Receiving; 2 - Enter the brush; 3 - Output from the brush; 4 - Enter the treadmill, 5 - Wake Fork; 6 Enter Classification; 7 - Output classification.

ARNAL et al. (2002) relataram que, em avaliação de linhas de beneficiamento para caqui "Rojo Brilhante", os valores máximos encontrados foram de $55 \mathrm{G}$ na saída da linha. Todavia, no presente trabalho, valores superiores a $50 \mathrm{G}$ foram encontrados em pontos de transferência na maioria das linhas avaliadas, com exceção da linha A, que apresentou valores inferiores. Os pontos de transferência com maiores valores em aceleração variaram entre os equipamentos observados, mas observa-se tendência para valores mais altos de aceleração na etapa de recebimento e na transferência para o polimento. Altos valores em aceleração também foram encontrados na etapa de recebimento para tomate de mesa (FERREIRA et al., 2005) e para cítrus (FERREIRA et al., 2006). Como na maioria das situações essa etapa é realizada manualmente, o controle da força utilizada é precário, demonstrando variações e passível de altos valores. Nos pontos de transferência, os valores encontrados ocorrem principalmente devido à queda em superfícies rígidas e alturas 
excessivas. A utilização de superfícies protetoras, em geral de baixo custo, aliada à redução na altura da queda nos pontos de transferência, pode reduzir bastante a magnitude dos impactos (MAGALHÃES et al., 2007).

\section{Efeito do impacto de queda nas qualidades físico-químicas do caqui "Rama Forte"}

Verificou-se que, no primeiro dia, os frutos do tratamento-controle apresentaram a menor perda de massa, enquanto, para os frutos submetidos à queda de 10 e $30 \mathrm{~cm}$, foram observados os maiores valores (Tabela 1). Os frutos derrubados a $20 \mathrm{~cm}$ tiveram valor intermediário entre os extremos. No terceiro dia, não houve diferença estatística entre os tratamentos. No sexto dia, os frutos do tratamento-controle e da queda de $10 \mathrm{~cm}$ apresentaram os menores valores de perda de massa, comparativamente aos frutos que sofreram impactos a $20 \mathrm{~cm}$ e a $30 \mathrm{~cm}$. Observa-se, portanto, crescente aumento na perda de massa durante o armazenamento, em especial para frutos submetidos a maiores alturas de queda.

MAGALHÃES \& FERREIRA (2006) constataram que tomates que passaram por protótipo de linha de beneficiamento em laboratório e sofreram queda de $30 \mathrm{~cm}$ sobre superfície de caixa plástica tiveram $3,46 \%$ de perda de massa após 8 dias em armazenamento a $23{ }^{\circ} \mathrm{C}$, causando maiores injúrias internas. Com a redução da altura de queda para $10 \mathrm{~cm}$, a perda de massa foi de 2,63\%. O ferimento e especialmente o amassamento conduzem ao acréscimo na atividade respiratória e aumento na perda de matéria seca e evaporação de água (CHITARRA \& CHITARRA, 2005; SOUZA et al., 2003). GUTIERREZ (2005) relata que a perda de massa é bom instrumento de medida do efeito do manejo de pós-colheita de frutos para a determinação dos pontos críticos da pós-colheita por técnicos de campo e produtores, sendo medida muito simples e rápida e de baixo custo.

TABELA 1. Perda de massa, expressa em percentagem, de caquis "Rama Forte" submetidos a diferentes alturas de queda, e classificado pelo produtor após 1; 3 e 6 dias de armazenamento. 'Rama Forte' persimmons mass loss in percentage submitted to different drop heights, and classified by the producer after 1; 3 and 6 days of storage.

\begin{tabular}{clll}
\hline \multirow{2}{*}{ Tratamentos } & \multicolumn{3}{c}{ Dias de Armazenamento } \\
\cline { 2 - 4 } & 1 & 3 & 6 \\
\hline \multirow{2}{*}{ Controle } & $0,43 \mathrm{a} *$ & $1,52 \mathrm{a}$ & $3,19 \mathrm{a}$ \\
& $0,07 * *$ & 0,16 & 0,31 \\
\hline \multirow{2}{*}{$10 \mathrm{~cm}$} & $0,50 \mathrm{~b}$ & $1,57 \mathrm{a}$ & $3,22 \mathrm{a}$ \\
& 0,08 & 0,24 & 0,47 \\
\hline \multirow{2}{*}{$20 \mathrm{~cm}$} & $0,48 \mathrm{ab}$ & $1,48 \mathrm{a}$ & $3,08 \mathrm{~b}$ \\
& 0,08 & 0,21 & 0,40 \\
\hline \multirow{2}{*}{$30 \mathrm{~cm}$} & $0,50 \mathrm{~b}$ & $1,52 \mathrm{a}$ & $3,37 \mathrm{c}$ \\
& 0,09 & 0,22 & 0,41 \\
\hline *Letras diferentes minúsculas significam diferença estatística na mesma coluna, com 95\% de confiança.
\end{tabular}

$\mathrm{Na}$ Tabela 2, são apresentados os teores de sólidos solúveis e $\mathrm{pH}$ e, na Tabela 3, a acidez titulável dos caquis submetidos a três alturas de quedas diferentes, durante o armazenamento. Não houve diferença nos teores de sólidos solúveis entre os frutos dos tratamentos durante a armazenagem, exceto no primeiro dia após o ensaio de queda, quando o maior valor foi do tratamento-controle e o menor foi dos frutos que sofreram queda de $30 \mathrm{~cm}$. Não houve diferença nos valores de $\mathrm{pH}$ e nos teores de acidez titulável entre os tratamentos durante o armazenamento. MAGALHÃES \& FERREIRA (2006), avaliando tomates submetidos a queda em três diferentes superfícies receptoras e três alturas de queda, não observaram diferença estatística ( $>0,05)$ para acidez titulável, pH e vitamina C. Por sua vez, DURIGAN et al. (2005) observaram para lima 
ácida, que frutos submetidos a impacto demonstravam, após armazenamento por 9 dias, maior perda de massa e redução significativa nos teores de sólidos solúveis, acidez titulável e ácido ascórbico do que frutos intactos.

TABELA 2. Teores de sólidos solúveis ( $\left.{ }^{\circ} \mathrm{Brix}\right)$ e $\mathrm{pH}$ de caquis "Rama Forte" submetidos diferentes alturas de queda após 1; 3 e 6 dias de armazenamento. Soluble solids ( ${ }^{\circ} \mathbf{B r i x}$ ) and pH of 'Rama Forte' persimmons submitted to different drop heights after 1; 3 and 6 days of storage.

\begin{tabular}{|c|c|c|c|c|c|c|c|c|}
\hline \multirow{2}{*}{ Tratamentos } & \multicolumn{4}{|c|}{ Sólidos Solúveis ( ${ }^{\circ}$ Brix) } & \multicolumn{4}{|c|}{$\mathrm{pH}$} \\
\hline & 0 & 1 & 3 & 6 & 0 & 1 & 3 & 6 \\
\hline \multirow{2}{*}{ Controle } & $19,99 \mathrm{a}^{*}$ & $20,07 \mathrm{~b}$ & $17,66 \mathrm{a}$ & $17,16 \mathrm{a}$ & $5,45 \mathrm{a}$ & $5,64 \mathrm{a}$ & $5,65 \mathrm{a}$ & $5,50 \mathrm{a}$ \\
\hline & $0,80 * *$ & 1,09 & 2,01 & 0,76 & 0,03 & 0,06 & 0,13 & 0,20 \\
\hline \multirow{2}{*}{$10 \mathrm{~cm}$} & $19,99 \mathrm{a}$ & $17,82 \mathrm{ab}$ & $17,41 \mathrm{a}$ & $17,91 \mathrm{a}$ & $5,45 \mathrm{a}$ & $5,66 \mathrm{a}$ & $5,60 \mathrm{a}$ & $5,62 \mathrm{a}$ \\
\hline & 0,80 & 1,75 & 0,52 & 1,42 & 0,03 & 0,06 & 0,10 & 0,11 \\
\hline \multirow{2}{*}{$20 \mathrm{~cm}$} & $19,99 \mathrm{a}$ & $17,41 \mathrm{ab}$ & $18,91 \mathrm{a}$ & $17,66 \mathrm{a}$ & $5,45 \mathrm{a}$ & $5,62 \mathrm{a}$ & $5,65 \mathrm{a}$ & $5,54 \mathrm{a}$ \\
\hline & 0,80 & 1,01 & 0,8 & 1,76 & 0,03 & 0,01 & 0,19 & 0,16 \\
\hline \multirow{2}{*}{$30 \mathrm{~cm}$} & $19,99 \mathrm{a}$ & $16,16 \mathrm{a}$ & $18,41 \mathrm{a}$ & $17,32 \mathrm{a}$ & $5,45 \mathrm{a}$ & $5,64 \mathrm{a}$ & $5,73 \mathrm{a}$ & $5,52 \mathrm{a}$ \\
\hline & 0,80 & 0,63 & 0,29 & 0,50 & 0,03 & 0,03 & 0,08 & 0,06 \\
\hline
\end{tabular}

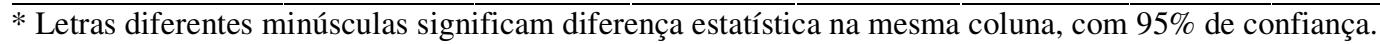

**Desvio-padrão.

TABELA 3. Acidez titulável, em ácido málico $\left(\mathrm{g} 100 \mathrm{~mL}^{-1}\right)$ de caquis "Rama Forte" submetidos a diferentes alturas de queda após 1; 3 e 6 dias de armazenamento. Tritable Acidity in malic acid $\left(\mathrm{g} 100 \mathrm{ml}^{-1}\right)$ of 'Rama Forte' persimmons submitted to different drop heights after 1; 3 and 6 days of storage.

\begin{tabular}{cllll}
\hline \multirow{2}{*}{ Tratamentos } & \multicolumn{4}{c}{ Acidez Titulável $\left(\mathrm{g} 100 \mathrm{~mL}^{-1}\right)$} \\
\cline { 2 - 5 } & 0 & $0,18 \mathrm{a}$ & 3 & 6 \\
\hline \multirow{2}{*}{ Controle } & $0,20 \mathrm{a}^{*}$ & 0,02 & $0,33 \mathrm{a}$ & $0,18 \mathrm{a}$ \\
& $0,01^{* *}$ & $0,19 \mathrm{a}$ & 0,09 & 0,02 \\
\hline \multirow{2}{*}{$10 \mathrm{~cm}$} & $0,20 \mathrm{a}$ & 0,02 & $0,23 \mathrm{a}$ & $0,19 \mathrm{a}$ \\
& 0,01 & $0,17 \mathrm{a}$ & $0,23 \mathrm{a}$ & 0,01 \\
\hline \multirow{2}{*}{$20 \mathrm{~cm}$} & $0,20 \mathrm{a}$ & 0,01 & 0,04 & 0,01 \\
\hline \multirow{2}{*}{$30 \mathrm{~cm}$} & 0,01 & $0,18 \mathrm{a}$ & $0,2 \mathrm{a}$ & $0,22 \mathrm{a}$ \\
& $0,20 \mathrm{a}$ & 0,01 & 0,01 & 0,02 \\
\hline
\end{tabular}

* Letras diferentes minúsculas significam diferença estatística na mesma coluna, com $95 \%$ de confiança.

**Desvio-padrão.

Em relação à aparência externa, os tratamentos não se diferenciaram em relação à luminosidade (L) até o primeiro dia (Tabela 4). Porém, os frutos com menores valores de L, que foram submetidos a maiores quedas, tiveram escurecimento acentuado durante armazenamento. A partir do terceiro dia, frutos submetidos a queda de $20 \mathrm{~cm}$ e $30 \mathrm{~cm}$ apresentaram tendência de redução dos valores de $\mathrm{L}$ em relação aos frutos do tratamento-controle e $10 \mathrm{~cm}$. Assim, verifica-se que os frutos que sofreram as maiores quedas apresentaram coloração da casca mais escura em relação aos frutos sem impactos e aos que caíram a $10 \mathrm{~cm}$, indicando a influência da queda na aparência dos frutos. 
TABELA 4. Valores de luminosidade (L) de caquis "Rama Forte" submetidos a diferentes alturas de queda após 1; 3 e 6 dias de armazenamento. Lightness values (L) of 'Rama Forte' persimmons submitted to different drop heights after 1; 3 and 6 days of storage.

\begin{tabular}{ccccc}
\hline \multirow{2}{*}{ Tratamentos } & \multicolumn{4}{c}{ Dias } \\
\cline { 2 - 5 } & 0 & 1 & 3 & 6 \\
\hline \multirow{2}{*}{ Controle } & $55,41 \mathrm{a}^{*}$ & $53,59 \mathrm{a}$ & $49,26 \mathrm{bc}$ & $44,13 \mathrm{ab}$ \\
& $1,63^{* *}$ & 1,78 & 2,89 & 5,12 \\
\hline \multirow{2}{*}{$10 \mathrm{~cm}$} & $55,28 \mathrm{a}$ & $53,7 \mathrm{a}$ & $49,49 \mathrm{c}$ & $46,52 \mathrm{~b}$ \\
& 1,72 & 1,79 & 2,91 & 5,01 \\
\hline \multirow{2}{*}{$20 \mathrm{~cm}$} & $55,49 \mathrm{a}$ & $53,49 \mathrm{a}$ & $47,25 \mathrm{a}$ & $42,37 \mathrm{a}$ \\
& 2,48 & 2,85 & 3,58 & 5,45 \\
\hline \multirow{2}{*}{$30 \mathrm{~cm}$} & $55,55 \mathrm{a}$ & $53,6 \mathrm{a}$ & $47,54 \mathrm{ab}$ & $42,05 \mathrm{a}$ \\
& 2,95 & 2,89 & 3,45 & 4,43 \\
\hline
\end{tabular}

* Letras diferentes minúsculas significam diferença estatística na mesma coluna, com 95\% de confiança.

**Desvio-padrão.

No primeiro dia, o tratamento-controle apresentou menores valores para chroma, os tratamentos 10 e $20 \mathrm{~cm}$ os maiores, e o tratamento $30 \mathrm{~cm}$ valor intermediário (Tabela 5). A partir do terceiro dia de armazenagem, frutos submetidos a queda de 20 e $30 \mathrm{~cm}$ apresentaram menores valores, não estatisticamente diferentes entre si. O tratamento $10 \mathrm{~cm}$ demonstrou maiores médias, não significativamente diferentes do controle. Portanto, a partir do terceiro dia, as quedas maiores que $10 \mathrm{~cm}$ apresentaram decréscimo mais acentuado em chroma devido à redução da saturação, indicando diminuição da intensidade da cor, associada ao escurecimento da casca. Frutos submetidos a quedas possuem perda na qualidade da aparência externa. DURIGAN et al. (2005) relatam maior perda da coloração verde da casca em lima ácida submetida a impacto.

TABELA 5. Valores de cromaticidade ou saturação da cor (Chroma) de caquis "Rama Forte" submetidos a diferentes alturas de queda após 1; 3 e 6 dias de armazenamento.

Chromaticity values or color saturation (Chroma) of 'Rama Forte' persimmons submitted to different drop heights after 1; 3 and 6 days of storage.

\begin{tabular}{ccccc}
\hline \multirow{2}{*}{ Tratamentos } & \multicolumn{4}{c}{ Dias } \\
\cline { 2 - 5 } & 0 & 1 & 3 & 6 \\
\hline \multirow{2}{*}{ Controle } & $65,07 \mathrm{a} *$ & $64,29 \mathrm{a}$ & $58,91 \mathrm{ab}$ & $47,19 \mathrm{ab}$ \\
& $1,75^{* *}$ & 2,04 & 3,86 & 9,89 \\
\hline \multirow{2}{*}{$10 \mathrm{~cm}$} & $64,95 \mathrm{a}$ & $65,83 \mathrm{~b}$ & $60,23 \mathrm{~b}$ & $54,31 \mathrm{~b}$ \\
& 1,86 & 1,69 & 3,67 & 8,96 \\
\hline \multirow{2}{*}{$20 \mathrm{~cm}$} & $64,48 \mathrm{a}$ & $65,82 \mathrm{~b}$ & $56,96 \mathrm{a}$ & $44,79 \mathrm{a}$ \\
& 2,73 & 3,06 & 5,28 & 11,03 \\
\hline \multirow{2}{*}{$30 \mathrm{~cm}$} & $65,81 \mathrm{a}$ & $64,4 \mathrm{ab}$ & $57,14 \mathrm{a}$ & $44,09 \mathrm{ab}$ \\
& 2,81 & 3,04 & 5,03 & 9,70 \\
\hline \multirow{2}{*}{ * Letras diferentes minúsculas significam diferença estatística na mesma coluna, com 95\% de confiança. }
\end{tabular}

\section{Danos internos}

No caso do caqui "Rama Forte", os danos internos -como consequência do impacto de quedaficaram evidenciados pelo escurecimento da região atingida pela queda, seguida pelo "amolecimento" do fruto, que corresponde à ruptura de membranas e consequente perda de firmeza dos frutos (Figuras 4; 5 e 6). O dano físico não é visível externamente, porém, ao serem cortados, é possível observar o efeito do impacto na parte interna. Em alguns frutos, a mudança de cor da polpa em decorrência do amadurecimento pode disfarçar o dano, ficando menos evidente. Verificou-se, 
também, que a posição do fruto no momento da queda tem influência sobre a magnitude do dano interno: quanto menor for a área de contato, maior é a pressão sobre essa área, aumentando, assim, a magnitude do impacto e, consequentemente, o dano interno correspondente à ruptura de membranas celulares. Dessa forma, mesmo quedas relativamente pequenas, como $10 \mathrm{~cm}$, podem causar danos, se o impacto for localizado.

No terceiro e sexto dias de armazenamento, as frutas que sofreram queda de $10 \mathrm{~cm}$ não apresentaram nenhum dano interno visível, enquanto nos caquis que sofreram queda de $20 \mathrm{~cm}$, no terceiro dia, havia $42,9 \%$ de frutos com danos internos, e no sexto dia foram $44,4 \%$. No terceiro dia, os frutos que caíram a $30 \mathrm{~cm}$ de altura tiveram a maior percentagem de danos, de $88,9 \%$. Contudo, no sexto dia, esse tratamento apresentou percentagem menor que os frutos que caíram a $20 \mathrm{~cm}$, com 28,6\% dos caquis com danos internos.

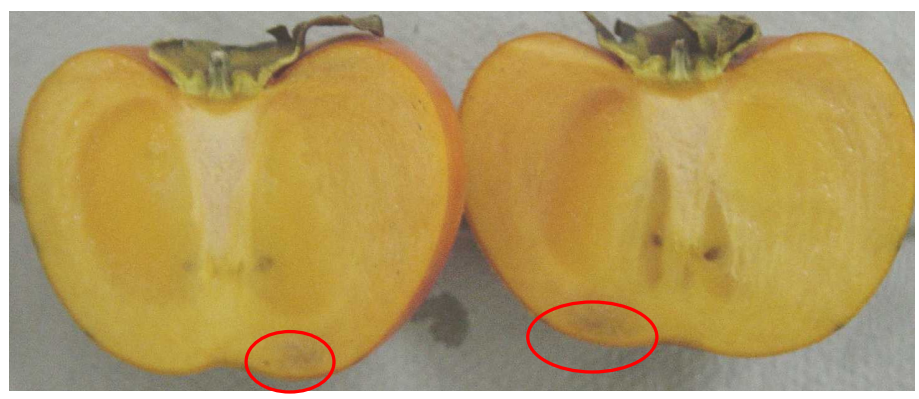

FIGURA 4. Dano interno em caqui "Rama Forte" submetido a queda de $10 \mathrm{~cm}$ de altura. Internal damage in 'Rama Forte' persimmon submitted to the $10 \mathrm{~cm}$ fall.

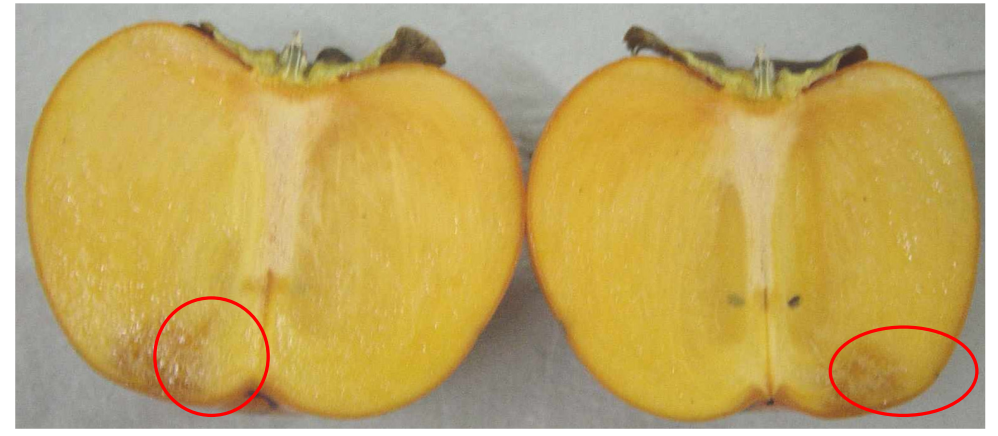

FIGURA 5. Dano interno em caqui "Rama Forte" submetido a queda de $20 \mathrm{~cm}$ de altura. Internal damage in 'Rama Forte' persimmon submitted to the $20 \mathrm{~cm}$ fall.

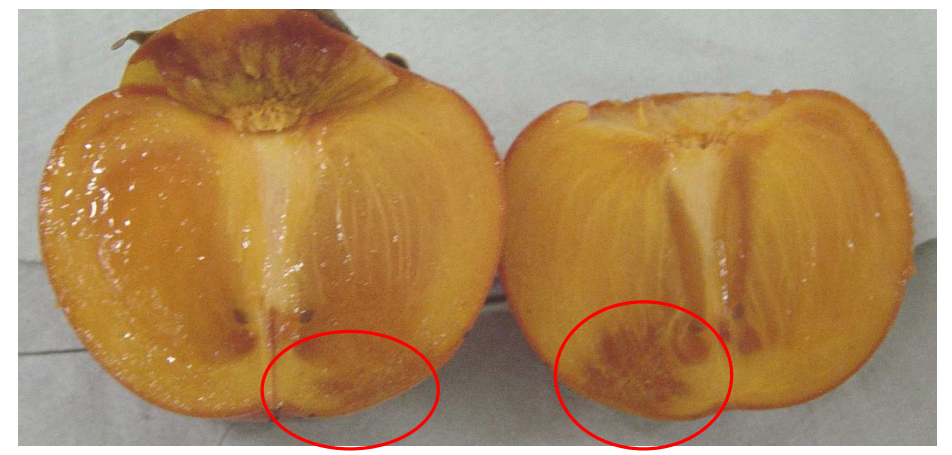

FIGURA 6. Dano interno em caqui "Rama Forte" submetido a queda de $30 \mathrm{~cm}$ de altura. Internal damage in 'Rama Forte' persimmon submitted to the $30 \mathrm{~cm}$ fall. 
As diferentes alturas de queda não causaram diferenças estatísticas na remoção da adstringência em nenhum dia da avaliação (Tabela 7). Entretanto, no sexto dia, os tratamentoscontrole e queda de $10 \mathrm{~cm}$ apresentaram alguns frutos adstringentes (nota 4), enquanto caquis dos tratamentos de queda de 20 e $30 \mathrm{~cm}$ estavam sem adstringência (notas próximas a 1). Uma das possíveis razões para isso pode estar relacionada à dimensão do dano físico. Medições futuras podem ser realizadas no local exato da queda.

TABELA 7. Médias de notas de remoção de adstringência de caquis "Rama Forte" submetidos a diferentes alturas de queda após 1; 3 e 6 dias de armazenamento. Removal astringency grade averages of 'Rama Forte' persimmons submitted to different drop heights after 1; 3 and 6 days of storage.

\begin{tabular}{clll}
\hline \multirow{2}{*}{ Tratamentos } & \multicolumn{3}{c}{ Dias } \\
\cline { 2 - 4 } & 1 & 3 & 6 \\
\hline \multirow{2}{*}{ Controle } & $4,00 \mathrm{a}^{*}$ & $1,33 \mathrm{a}$ & $1,78 \mathrm{a}$ \\
& $0,00 * *$ & 0,71 & 1,30 \\
\hline \multirow{2}{*}{10} & $4,00 \mathrm{a}$ & $1,56 \mathrm{a}$ & $1,89 \mathrm{a}$ \\
& 0,00 & 0,73 & 1,36 \\
\hline \multirow{2}{*}{20} & $4,00 \mathrm{a}$ & $2,14 \mathrm{a}$ & $1,44 \mathrm{a}$ \\
& 0,00 & 0,90 & $1,00 \mathrm{a}$ \\
\hline \multirow{2}{*}{30} & $4,00 \mathrm{a}$ & $1,89 \mathrm{a}$ & 0,00 \\
\hline * Letras diferentes minúsculas significam diferença estatística na mesma coluna, com $95 \%$ de confiança. \\
**Desvio-padrão.
\end{tabular}

\section{CONCLUSÕES}

Caquis "Rama Forte" submetidos ao beneficiamento e à classificação em unidades de beneficiamento estão sujeitos à alta magnitude em impacto, dependendo do ponto de transferência e da força utilizada. Alterações são necessárias, como a utilização de protetores de impacto e diminuição na altura de queda nos pontos de transferência. Nos ensaios em laboratório, determinou-se que a altura máxima de queda é de $10 \mathrm{~cm}$. Nessa condição, a perda de massa não é diferente de um fruto sem dano depois de seis dias do impacto, além de não prejudicar a coloração da casca. Pelos resultados obtidos, é possível observar uma correlação entre maior incidência de danos físicos e modificações na coloração externa dos frutos. Maiores alturas de queda indicam perda progressiva na qualidade do fruto.

\section{AGRADECIMENTOS}

À FAPESP, pelo suporte financeiro (processo 03/06445-2 - Projeto Políticas Públicas).

\section{REFERÊNCIAS}

ARNAL, L.; RIO, M.A. del; VENDRELL, M. Effects of packing line operations on some quality attributes of persimmon fruit cv. "Rojo Brillante". In: MEDITERRANEAN SYMPOSIUM ON PERSIMMON, 2001, Faenza. Abstracts... 2002. p.113-117.

BAJEMA, R.W.; HYDE, G.M. Packing line bruise evaluation for 'Walla Walla' summer sweet onions. Transactions of the ASAE, St. Joseph, v.38, n.4, p.1.167-1.171, 1995.

BOLLEN, A.F. Technological innovations in sensors for assessment of postharvest mechanical handling systems. International Journal of Postharvest Technology and Innovation, Geneve, v.1, n.1, p.16-31, 2006. 
BROWN, G. K.; SCHULTE PASON, N. L.; TIMM, E. J.; BURTON, C. L.; MARSHALL, D. E. Apple packing line impact damage reduction. Applied Engineering in Agriculture, St. Joseph, v.6, n.6, p.759-764, 1990.

BRUSEWITZ, G.H.; McCOLLUM, T.G.; ZHANG, X. Impact bruise resistance of peaches. Transactions of the ASAE, St. Joseph, v.34, n.3, p.962-965, 1991.

CARVALHO, C.R.L.; MANTOVANI, D.M.; CARVALHO, P.R.N.; MORAES, R.M. Análises químicas de alimentos: manual técnico. Campinas: Biblioteca do ITAL, 1990. $121 \mathrm{p}$.

CHEN, P.; YAZDANI, R. Prediction of apple bruising due to impact on different surfaces. Transactions of the ASAE, St. Joseph, v.34, n.3, p. 956-961, 1991.

CHITARRA, M.I.F.; CHITARRA, A.B. Pós-colheita de frutos e hortaliças: fisiologia e manuseio. 2.ed. Lavras: ESAL/FAEFE, 2005. 785 p.

DAREZZO, H.M.; BENEDETTI, BC.; DELIZA, R.; CENCI, S.; GONÇALVES, E.B. Evaluation of quality attributes of fresh-cut lettuce (Lactuca sativa $\mathrm{L}$.) stored in different controlled atmospheres. Acta Horticulturae, Amsterdam, v.600, p.213-219, 2003.

DURIGAN, M.F.B.; MATTIUZ, B.; DURIGAN, J.F. Injúrias mecânicas na qualidade pós-colheita de lima ácida "Tahiti” armazenada sob condição ambiente. Revista Brasileira de Fruticultura, Jaboticabal, v.27, n.3, p.369-372, 2005.

FERREIRA, M.D.; FERRAZ, A.C.O.; FRANCO, A.T.O. Tomato packing lines studies with na instrumented sphere in Brazil. Acta Horticulturae, Amsterdam, v.682, p.1.753-1.760, 2005.

FERREIRA, M.D.; NETTO, L.H. Avaliação de processos nas linhas de beneficiamento e classificação de batatas. Horticultura Brasileira, Brasília, v.25, n.2, p.279-285, 2007.

FERREIRA, M.D.; SILVA, M.C.; CAMARGO, G.G.T.; AMORIM, L.; FISCHER, I.H. Pontos críticos de impacto em linhas de beneficiamento utilizadas para citros no Estado de São Paulo. Revista Brasileira de Fruticultura, Jaboticabal, v.28, n.3, p.523-525, 2006.

FLUCK, R.C.; HALSEY, L.H. Impact forces and tomato bruising. Florida Agricultural Experiment Station Journal Series, Gainesville, n.5.109, p.239-242, 1973.

GARCÍA-RAMOS, F.J.; ORTIZ-CAÑAVATE, J.; RUIZ-ALTISENT, M. Decelerator elements for ramp transfer points in fruit packing lines. Journal of Food Engineering, Ontario, v.59, n.4, p. 331337, 2003.

GAZIT, S.; LEVY, Y. Astringency and its removal in persimmon. Israel Journal of Agricultural Research, Rehovot, v.13, n.3, p.125-132, 1963.

GUTIERREZ, A.S.D. Danos mecânicos pós-colheita em pêssego fresco. 2005. 124 f. Tese (Doutorado em Produção Vegetal) - Escola Superior de Agricultura "Luiz de Queiroz", Universidade de São Paulo, Piracicaba, 2005.

HYDE, G.M.; BROWN, G.K.; TIMM, E.J.; ZHANG, W. Instrumented sphere evaluation of potato packing line impacts. Transactions of the ASAE, St. Joseph, v 35, n 1, p.65-69, 1992.

HYDE, G.M.; ZHANG, W. Apple bruising research update: packing line impact evaluations. Tree Fruit Postharvest Journal, Wenatchee, v.3, n.3, p.12-15, 1992.

IBGE. INSTITUTO BRASILEIRO DE GEOGRAFIA E ESTATÍSTICA. Produção Agrícola Municipal. Rio de Janeiro, 2004. Disponível em <www.sidra.ibge.gov.br>. Acesso em: 2 abr. 2007.

MAGALHÃES, A.M.; FERREIRA, M.D. Qualidade dos frutos de tomate de mesa quando submetidos a impacto por ocasião do beneficiamento. Horticultura Brasileira, Brasília, v.24, n.4, p.481-484, 2006. 
MAGALHÃES, A.M.; FERREIRA, M.D.; BRAUNBECK, O.A.; ESTEVON, M.V.R. Superfícies protetoras na diminuição de danos mecânicos em tomate de mesa. Ciência Rural, Santa Maria, v.37, n.3, p.878-881, 2007.

MANESS, N.O.; BRUSEWITZ, G.H.; McCOLLUM, T.G. Impact bruise resistance comparison among peach cultivars. HortScience, Alexandria, v.27, n.9, p.1.008-1.011, 1992.

MATTIUZ, B.H.; DURIGAN, J.F. Efeito de injúrias mecânicas no processo respiratório e nos parâmetros químicos de goiabas "Paluma" e "Pedro Sato". Revista Brasileira de Fruticultura, Jaboticabal, v.23, n.2, p.282-287, 2001.

MATTIUZ, B.H.; DURIGAN, J.F. Fisiologia e qualidade pós-colheita de goiabas "Paluma" e "Pedro Sato" submetidas a injúria mecânica por impacto. Revista Brasileira de Armazenamento, Viçosa - MG, v.28, n.1, p.46-50, 2003.

MENCARELLI, F.; MASSANTINI, R.; BOTONDI, R. Influence of impact surface and temperature on the ripening response of kiwifruit. Postharvest Biology and Technology, Amsterdam, v.8, n.3, p.165-177, 1996.

MILLER, W.M.; WAGNER, C. Florida citrus packingline studies with an instrumented sphere. Applied Engineering in Agriculture, St. Joseph, v.7, n.5, p.577-581, 1991.

MORETTI, C.L.; SARGENT, S.A.; HUBER, D.J.; CALBO, A.G.; PUSCHMANN, R. Chemical composition and physical properties of pericarp, locule, and placental tissues of tomatoes with internal bruising. Journal of the American Society for Horticultural Science, Alexandria, v.123, n.4, p.656-600, 1998.

SARGENT, S.A.; BRECHT, J.K.; ZOELLNER, J.J. Instrumented sphere impact analysis of tomato and bell pepper packing lines. Applied Engineering in Agriculture, St. Joseph, v.8, n.1, p.76-83, 1992.

SARGENT, S.A.; RITENOUR, M.A.; BRECHT, J.K. Handling, cooling and sanitation techniques for maintaining postharvest quality. Gainesville: Horticultural Sciences Department HS719, Florida Cooperative Extension Service, Institute of Food and Agricultural Sciences, University of Florida. 1999.

SATO, G.S.; ASSUMPÇÃO, R. Caqui: uma opção para a produção familiar no Estado de São Paulo. Instituto de Economia Agrícola, 2002. Disponível em: <www.iea.sp.gov.br>. Acesso em: 2 abr. 2007.

SILVA, P.R.; BAPTISTELLA, C.S.L.; FRANCISCO, V.L.F. S. A cultura do caqui em São Paulo. Instituto de Economia Agrícola, 2005. Disponível em: <www.iea.sp.gov.br>. Acesso em: 2 abr. 2007.

SOUZA, R.M.; HENZ, G.P.; PEIXOTO, J.R. Incidência de injúrias mecânicas em raízes de mandioquinha-salsa na cadeia de pós-colheita. Horticultura Brasileira, Brasília, v.21, n.4, p.712718, 2003.

TREVISAN, R.; GONCALVES, E.D.; COUTINHO, E.F. Qualidade de pêssegos em pomares conduzidos de forma convencional e integrada. Ciência Rural, Santa Maria, v.34, n.6, p.1.7471.751, 2004. Disponível em: <http://www.scielo.br/scielo.php?script=sci_arttext\&pid=S010384782004000600012\&lng=en\&nrm=iso >. Acesso em: 21 maio 2007.

ZAPP, H.R.; EHLERT, S.H.; BROWN, G.K.; ARMSTRONG, P.R.; SOBER, S. Advanced instrumented sphere (IS) for impact measurements. Transactions of the ASAE, St. Joseph, v.33, n.3, p.955-960, 1990. 